\title{
Advances in conceptualizing and measuring stigma among people living with HIV
}

Project SOAR

Follow this and additional works at: https://knowledgecommons.popcouncil.org/departments_sbsr-hiv

Part of the Health Services Research Commons How does access to this work benefit you? Let us know!

\section{Recommended Citation}

Project SOAR. 2021. "Advances in conceptualizing and measuring stigma among people living with HIV," Learnings from Project SOAR, Synthesis Brief. Washington, DC: Population Council. 


\section{Advances in Conceptualizing and Measuring Stigma Among People Living with HIV}

\section{Learnings from Project SOAR}

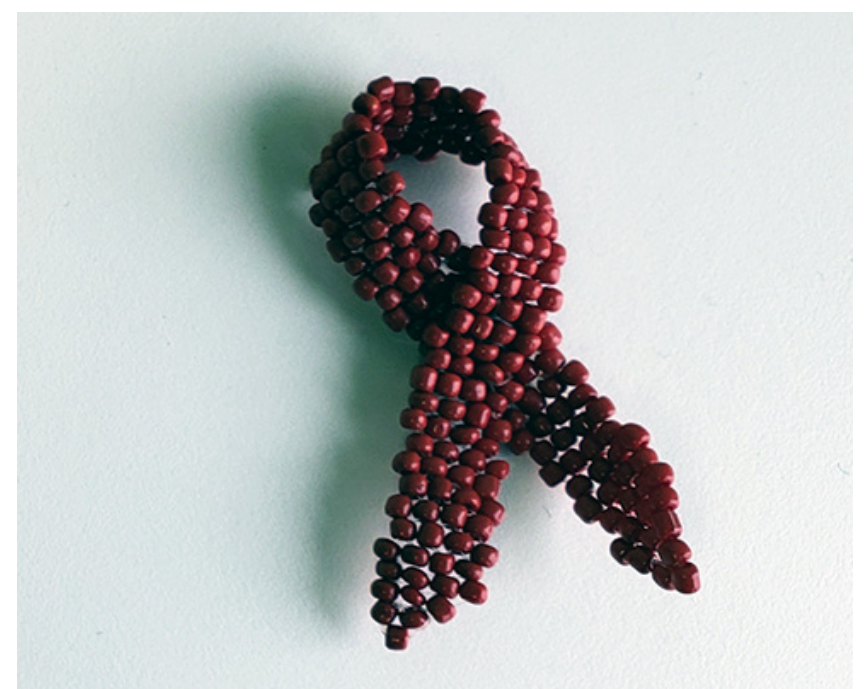

Stigma, defined as "the co-occurrence of labeling, stereotyping, separation, status loss, and discrimination in a context in which power is exercised," ${ }^{1}$ has a negative impact on the health of people living with HIV (PLHIV), contributing to psychosocial stress, coercion and violence, job loss, and social exclusion. ${ }^{2}$ Widely recognized as a barrier to accessing HIV care, it is paramount that programs and policies are designed to mitigate HIV-related stigma to achieve the UNAIDS' 90-90-90 goals.

Project SOAR recognizes the importance of addressing stigma to control the HIV epidemic through testing, treatment, and viral suppression. Drawing on its extensive portfolio, Project SOAR has contributed to the evidence base and catalyzed research use by conceptualizing and measuring stigma among PLHIV.

This brief highlights findings from two studies across four countries (see map on next page for additional detail) that examined:

\section{KEY RECOMMENDATIONS}

1. To effectively reach marginalized populations, HIV-related programs and policies must address intersectional stigmas attributable to HIV status and structural factors, including key population status. The revised People Living with HIV (PLHIV) Stigma Index 2.0 effectively captures stigma experiences due to HIV status, as well as stigma attributed to membership in marginalized social groups such as sexual minorities.

2. Interventions to improve the health and well-being of PLHIV should emphasize cultivating and strengthening resilience. The novel People Living with HIV Resilience Scale performed well in capturing the extent to which one's ability to meet various needs and important life goals has been affected by having HIV. The Resilience Scale could be used to evaluate and strengthen interventions around building coping skills to manage the effects of this stigma, enhancing stress management, and increasing social support.

- Intersectional stigma among key populations, including impacts on HIV outcomes; and

- The importance of measuring and promoting resilience to combat stigma. 
Dominican Republic Implementation of PLIHV Stigma Index 2.0

PLIHV Stigma Index Pilot

Senegal

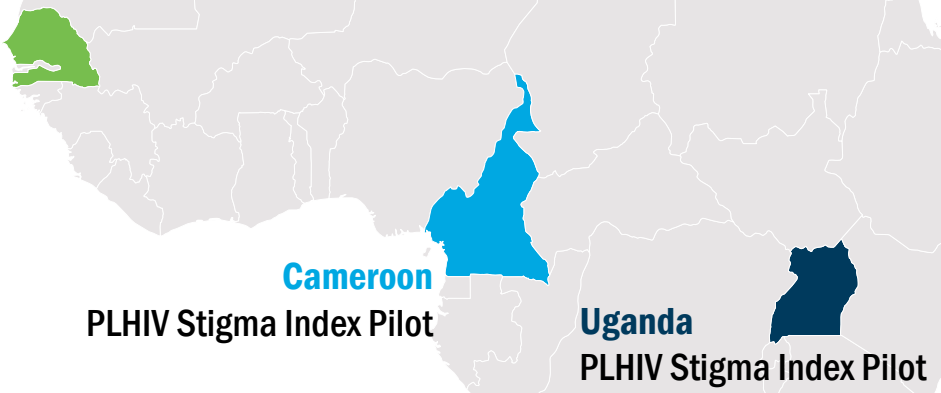

\section{ABOUT}

Project SOAR (Supporting Operational AIDS Research), was a 6-year USAID-funded implementation science initiative with over 70 activities in 21 countries. The Population Council led Project SOAR in collaboration with Avenir Health, Elizabeth Glaser Pediatric AIDS Foundation (EGPAF), Johns Hopkins University, Palladium, and The University of North Carolina at Chapel Hill.

\section{Research Utilization}

Project SOAR implemented a systematic and proactive research utilization (RU) approach to advance translation of research results into evidence-informed practice. This $\mathrm{RU}$ approach supported inclusion of end data users in the research process from inception to dissemination and fostered data-driven policy and program change.

\section{Series}

"Learnings from Project SOAR" seeks to highlight key results, recommendations, and examples of research impact across this 6-year, 21-country initiative.

The series includes five thematic briefs focused on biomedical prevention interventions, HIV testing services, HIV treatment strategies, stigma, and research utilization and capacity strengthening.

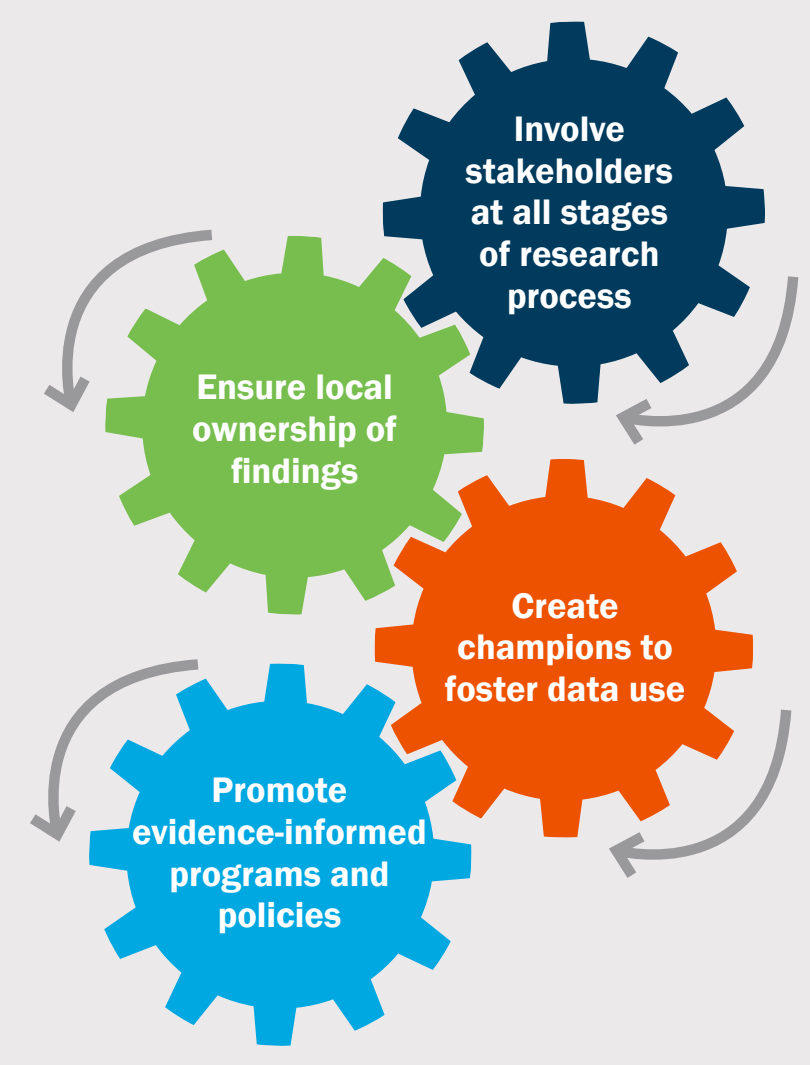

2 | Advances in conceptualizing and measuring stigma among people living with HIV 


\section{INTERSECTIONAL STIGMA AMONG KEY POPULATIONS LIVING WITH HIV}

Overview: While many people Iiving with HIV
experience multiple intersecting stigmas-such
as those due to HIV status, gender identity,
sexual orientation, and race or ethnicity-
to date, the study of intersectional stigma
has been largely qualitative. Project SOAR
researchers, community members, and
advocates took on the challenge of capturing
intersectional stigma quantitatively when they
updated the PLHIV Stigma Index and pilot
tested the expanded measures in Cameroon,
Senegal, Uganda, and the Dominican Republic.

Results summary: Key populations are

situated at the intersection of HIV-related

stigma and stigma associated with their

identities, occupations, and behaviors, often

experiencing multiple forms of marginalization.

Key populations living with HIV attributed

stigma experiences to their membership in

marginalized communities, in addition to

stigma due to their seropositive HIV status.

In quantitatively examining intersectional

stigma, the PLHIV Stigma Index 2.0 creates

opportunities to generate local information

needed to inform stigma reduction

interventions, monitor progress over time, and

advocate for the rights of PLHIV. all three countries, a total of 1,207 people living with HIV participated in the pilot study (approximately 400 per country). About half in each country were members of key populations, including female and male sex workers, men who have sex with men (MSM), people who use drugs, and transgender men and women.

The pilot study found that $\mathbf{3} 3$ percent of key population members living with HIV in Senegal experienced at least one form of stigma compared to 18 percent of other respondents. In Uganda, approximately 20 percent of respondents reported at least one experience of stigma and discrimination in a HIV healthcare setting in the previous year. However, MSM and transgender males and females experienced higher levels of stigma and discrimination than other groups. The study also documented high rates of violence against key populations living with HIV. For example, forced sex was reported by 53 percent of sex workers in Cameroon, 40 percent in Senegal, and 32 percent in Uganda. For MSM, 30

Updating the People Living with HIV Stigma Index

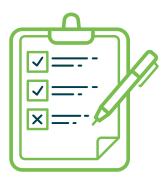

Survey implemented by and for PLHIV to document stigma and discrimination and advocate for supportive programs and policies

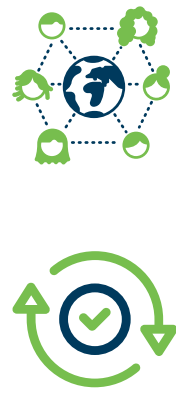

Developed in 2008 by the Global Network of PLHIV (GNP+), International Community of Women Living with HIV (ICW), International Planned Parenthood Federation (IPPF), and UNAIDS

Updated in 2017 (PLHIV Stigma Index 2.0) through an international partnership including GNP+, ICW, UNAIDS, Project SOAR, and other stigma researchers

SOAR PLHIV Stigma Index 2.0 Pilot Country Research Partners population members living with HIV experienced heightened levels of stigma compared to other populations and high rates of violence.

SOAR researchers provided technical assistance to local partners in Cameroon, Senegal, and Uganda to pilot test an expanded PLHIV Stigma Index 2.0, including questions on intersectional stigma. Across

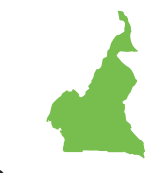

Cameroon

Metabiota \& Récap+

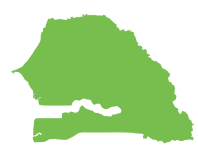

Senegal

Enda Santé \& RNP+

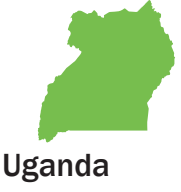

National Forum for People Living with HIV 
percent in Senegal, 23 percent in Cameroon, and 16 percent in Uganda reported forced sex. ${ }^{3}$

In the Dominican Republic, compared to nonkey populations, female sex workers reported higher levels of harassment and stigma due to their HIV status.

Following the successful pilot of the revised PLHIV Stigma Index 2.0 described above, the updated survey was translated to Spanish and implemented in the Dominican Republic, where 891 PLHIV, more than half of whom were members of key populations (216 FSWs, 154 MSM, and 90 of Haitian descent), participated in a SOAR-led study. Compared to other women living with HIV, FSWs reported significantly higher levels of harassment due to their HIV status. For example, a larger proportion of FSWs had experienced at least one type of verbal or physical harassment on account of their HIV status. The most frequently reported type of harassment experienced by FSWs was verbal harassment, experienced by onequarter of respondents. In addition, FSWs reported more internalized stigma, "real or imagined fear of discrimination," ${ }^{4}$ than non-key populations.

FSWs were also more likely to report experiencing stigma in HIV and non-HIV healthcare services than other women living with HIV. Nearly one-fifth of FSWs reported at least one stigmatizing experience in HIV-specific healthcare services, compared to 13 percent among other women living with HIV. Similarly, 19 percent of FSWS had at least one stigmatizing experience in non-HIV-specific healthcare services, compared to 11 percent of other women living with HIV. Notably, despite their marginalized status as racial and ethnic minorities, respondents of Haitian descent reported lower levels of social exclusion or harassment, compared to those who were not of Haitian descent. ${ }^{5}$

\section{In Cameroon, HIV positive members of key populations attributed experiences of stigma to their marginalized status.}

In some cases, seropositive key population individuals reported stigma experiences that they attributed to their membership in these marginalized communities, which, depending on the context and community, can be more pronounced than stigma due to their HIV status. For example, in the pilot test of the PLHIV Stigma Index 2.0 in Cameroon, 66 percent of seropositive MSM reported experiencing verbal harassment on account of their HIV status. In addition to this stigma attributed to their seropositive status, 84 percent of MSM said they had experienced verbal harassment because they have sex with men. Conversely, 50 percent of FSWs reported discriminatory remarks by family members due to their seropositive status, and nearly 1 in 5 attributed this experience to their selling sex.

\section{Key finding: Impacts of stigma on HIV outcomes}

\section{In the Dominican Republic, researchers found mixed results when examining the relation- ship between specific experiences of stigma and HIV outcomes.}

In the aforementioned study in the Dominican Republic, key population communities reported lower levels of knowing they were virally suppressed and were more likely to have missed an ART dose in the past year, compared to other PLHIV. However, researchers found mixed results when examining the relationship between specific experiences of stigma and HIV outcomes.

MSM who reported a greater number of stigma experiences in HIV-specific services had a significantly lower odds of knowing they had an undetectable viral load, compared to men who were not MSM. Higher internalized stigma scores were significantly associated with missing an ART dose among FSWs, compared to women who did not sell sex. For key population groups, experiencing stigma in either HIVspecific or non-HIV-specific healthcare services was not significantly associated with missing an ART dose. 


\section{THE IMPORTANCE OF MEASURING AND PROMOTING RESILIENCE TO COMBAT STIGMA}

\begin{abstract}
Overview: Networks of people living with HIV, advocates, and donors have recommended measuring the resilience of PLHIV in addition to experiences of stigma. In response, SOAR researchers developed the PLHIV Resilience Scale in 2017, which documents the extent to which one's ability to meet various needs and important life goals has been affected by having HIV, and evaluated the scale in three countries (Cameroon, Senegal, and Uganda).
\end{abstract}

Results summary: The new PLHIV Resilience Scale, included in the PLHIV Stigma Index 2.0, performed well in capturing the extent to which one's ability to meet various needs and important life goals has been affected by having HIV. A sizable minority of respondents reported this resilience in the face of HIV and those who were more resilient were more likely to be aware of human rights protections and less likely to report internalized stigma. Results highlight the importance of capturing positive, and not only negative, impacts of HIV status on individuals' wellbeing. This is a valuable tool that can be used to assess whether programs improve PLHIV resilience over time.

\section{Key findings}

Across Cameroon, Senegal, and Uganda, the Resilience Scale performed well in factor analyses, demonstrated very good reliability, and captured a range of experiences of resilience both within and between countries.

SOAR evaluated performance of the Resilience Scale with 1,207 PLHIV in Cameroon, Senegal and Uganda as part of pre-testing the draft PLHIV Stigma Index 2.0. The new Resilience Scale performed well, demonstrating very good reliability, and capturing a range of experiences of resilience both within and between countries. Further, significant associations between the scale and overall health, depression, and experience of stigma/discrimination support convergent validity. ${ }^{6}$

Within each country, individuals' composite scale scores (sum of all items) varied widely, suggesting that the scale can capture a diverse range of resilience experiences. In addition, each scale item exhibited a good range of responses: while a majority of respondents tended to answer "was not affected by my HIV status", a sizeable minority answered either "was positively affected" or "was negatively affected". It was surprising that most respondents answered "was not affected" for most items and a sizeable minority indicated they were positively affected by their HIV status. This could reflect improvements in HIV treatment and consequent decreases in HIVrelated morbidity, which may have reduced the extent to which having HIV is salient to different aspects of people's lives. This serves to further affirm the importance of capturing positive, and not only negative, effects of HIV status on individuals' wellbeing. ${ }^{6}$

\section{RESEARCH IMPACT SPOTLIGHT}

\section{People Living with HIV Stigma Index 2.0}

Following the successful revision and pilot of the People Living with HIV Stigma Index under SOAR in 2016-18, the revised instrument ("PLHIV Stigma Index 2.0") has now been implemented or has plans for implementation in over 30 countries. Access to the PLHIV Stigma Index can be requested through: https://www.stigmaindex. org/.

For more information, please visit Project SOAR. 


\section{REFERENCES}

1. Link, B. G. and J. C. Phelan. 2001. "Conceptualizing stigma," Annual Review of Sociology 27: 363-85. doi: 10.1146/annurev.soc.27.1.363

2. Rueda, S. et al. 2016. "Examining the associations between HIV-related stigma and health outcomes in people living with HIV/AIDS: a series of metaanalyses," BMJ Open 6(7): e011453. doi: 10.1136/ bmjopen-2016-011453

3. Project SOAR. 2018. "The People Living with HIV Stigma Index 2.0: Results from pilot testing in three countries," Project SOAR Results Brief. Washington, DC: Population Council. https://projsoar.org/wp-content/ uploads/2018/08/Stigmalndex_ResBrief.pdf

4. Brown, L., K. Macintyre, and L. Trujillo. 2003. "Interventions to reduce HIV/AIDS stigma: what have we learned?," AIDS Education Prevention 15(1): 49-69. doi:10.1521/aeap.15.1.49.23844

Suggested citation: Project SOAR. 2021. "Advances in conceptualizing and measuring stigma among people living with HIV," Learnings from Project SOAR Synthesis Brief. Washington, D.C.: Population Council.

For more information, please visit Project SOAR.
5. Yam, E. A. et al. 2020. "Stigma among key populations living with HIV in the Dominican Republic: experiences of people of Haitian descent, MSM, and female sex workers," AIDS 34(Suppl 1): S43-S52. doi: 10.1097/ QAD.0000000000002642

6. Gottert, A. et al. 2019. "The People Living with HIV (PLHIV) Resilience Scale: Development and validation in three countries in the context of the PLHIV Stigma Index," AIDS and Behavior 23(Suppl 2): 172-182. doi: 10.1007/ s10461-019-02594-6.
Project SOAR was a six-year (September 2014-January 2021) cooperative agreement funded by the United States President's Emergency Plan for AIDS Relief and the U. S. Agency for International Development (Agreement No. AID-OAA-A-14-00060). The contents of this brief are the sole responsibility of Project SOAR and Population Council and do not necessarily reflect the views of PEPFAR, USAID, or the United States Government.

Population Council led the Project SOAR consortium in collaboration with Avenir Health, Elizabeth Glaser Pediatric AIDS Foundation, the Johns Hopkins University, Palladium, and The University of North Carolina at Chapel Hill.
Project SOAR/Population Council

4301 Connecticut Avenue, NW, Suite 280

Washington, DC 20008

Tel: +12022379400

e-mail: ProjectSOAR@popcouncil.org projsoar.org

(C) Population Council, April 2021

Cover photo credit: Sherry Hutchinson/Population Council 